\title{
Comparison between measured divertor parameters in ASDEX Upgrade and SOLPS code solutions
}

\author{
A.V. Chankin ${ }^{a *}$, D.P. Coster ${ }^{\mathrm{a}}$, R. Dux ${ }^{\mathrm{a}}$, Ch. Fuchs ${ }^{\mathrm{a}}$, G. Haas ${ }^{\mathrm{a}}$, A. Herrmann ${ }^{\mathrm{a}}$, L.D. Horton ${ }^{\mathrm{a}}$, \\ A. Kallenbach ${ }^{\text {a }}$, M. Kaufmann ${ }^{\text {a }}$, A.S. Kukushkin ${ }^{\text {, }}$ K. Lackner ${ }^{\mathrm{a}}$, H.W. Müller ${ }^{\mathrm{a}}$, \\ J. Neuhauser ${ }^{\mathrm{a}}$, R. Pugno ${ }^{\mathrm{a}}$, M. Tsalas ${ }^{\mathrm{c}}$ and the ASDEX Upgrade Team \\ ${ }^{a}$ Max-Planck-Institut für Plasmaphysik, Boltzmannstr. 2, 85748 Garching, Germany \\ ${ }^{b}$ ITER International Team, Garching Joint Working Site, Boltzmannstr. 2, 85748 Garching, Germany \\ ${ }^{c}$ NCSR Demokritos, Greece
}

\begin{abstract}
Detailed modelling of the plasma edge of ASDEX Upgrade in Ohmic and H-mode discharges was performed with the SOLPS (B2-Eirene) code. A tendency of the code solutions to underestimate the divertor $\mathrm{T}_{\mathrm{e}}$ and overestimate the plasma density (thus, predicting cooler and denser divertor than in the experiment) has been reliably established. A possible cause of the observed discrepancies is the presence of a significant population of supra-thermal ions and electrons in the SOL and divertor, which can't be modelled by the fluid plasma code B2.
\end{abstract}

PACS: 52.55.Fa, 52.49.Hf, 52.65.-y, 52.25.Fi

JNM keywords: Plasma-Materials Interaction

PSI-17 keywords: ASDEX-Upgrade, B2-Eirene code, Divertor simulation, SOL transport

*Corresponding author address: Max-Planck-Institut für Plasmaphysik, Boltzmannstr. 2, 85748

Garching, Germany

*Corresponding author E-mail: Alex.Chankin@ipp.mpg.de

Presenting author: Dr A.V. Chankin Presenting author E-mail: Alex.Chankin@ipp.mpg.de 


\section{Introduction}

ASDEX Upgrade (AUG) has good diagnostic capabilities for measuring edge plasma parameters. Well resolved electron density, $\mathrm{n}_{\mathrm{e}}$, electron temperature, $\mathrm{T}_{\mathrm{e}}$, and ion temperature, $\mathrm{T}_{\mathrm{i}}$, profiles can be obtained at the outer midplane, covering outer core, separatrix and SOL regions. In the modelling described below, these midplane profiles were fitted by adjusting perpendicular transport coefficients using the SOLPS code package, which consists of the coupled fluid plasma code B2.5 and Monte-Carlo neutral code EIRENE. Comparison between simulated and measured plasma parameters in the divertor then enables benchmarking code solutions on the consistency with experiment. This strategy is explained in [1].

Typically, code solutions in the divertor agree within a factor of 2 with experimental measurements [2-4]. However, the tendency of the code solutions to underestimate the divertor $T_{e}$ and overestimate $n_{e}$, especially near the outer strike point position, has often been noticed. The present paper describes a comparison between SOLPS modelling and experiment based on two AUG shots: a standart AUG Ohmic shot is analysed in detail, while the previously modelled ELMy H-mode shot [1] is mostly reviewed, with only limited new information on further modelling being presented.

\section{Modelling of H-mode shot}

The results of the quasi-steady-state inter-ELM modelling of low density $\left(\bar{n}_{e}=5.8 \times 10^{19} \mathrm{~m}^{-3}\right)$ H-mode shot with $\mathrm{B}_{\mathrm{t}}=2 \mathrm{~T}, \mathrm{I}_{\mathrm{p}}=0.8 \mathrm{MA}$ and $5 \mathrm{MW}$ of NBI are described in [1]. This modelling revealed a significant discrepancy between simulated and measured parameters in the divertor, especially in the vicinity of the outer strike point (measured and simulated parameters at the inner target have not been compared in detail. The same applies to the Ohmic case described in 
the next section). The strongest direct indication of the discrepancy was a substantially higher, by factor 2.2, simulated peak $\mathrm{H}_{\alpha}$ radiation from the outer target than in the experiment. The simulated $\mathrm{H}_{\alpha}$ profile was also narrower than in the experiment. Extensive sensitivity studies, by varying input parameters in the code, were conducted. They included variation of: separatrix density within a certain allowable range, ion and electron parallel heat flux limiters, power sharing between ions and electrons, degree of ballooning of transport coefficients (by default, a rather arbitrarily chosen dependence $\sim 1$ / B is used in the modelling as a compromise between zero ballooning and a stronger ballooning, $\sim 1 / \mathrm{B}^{2}$, cases), the value of transport coefficients in the SOL and divertor, Helium content, models of the neutral description in the vacuum vessel, chemical sputtering coefficients, and prompt reflection coefficient of neutrals and ions from the target. The main discrepancy in the $\mathrm{H}_{\alpha}$ radiation profiles has not been eliminated.

Two possible explanations for the discrepancy were considered: some drawbacks (e.g. missing atomic or molecular reactions) of EIRENE which make the neutrals slower than in reality and result in narrow ionisation profiles, and the presence of a significant population of supra-thermal ions or electrons in the SOL and divertor plasma. The latter was related to low plasma collisionality in the SOL: in the code simulation, parameters at the separatrix midplane position were: $n_{e}=1.6 \times 10^{19} \mathrm{~m}^{-3}, \mathrm{~T}_{\mathrm{e}}=105 \mathrm{eV}, \mathrm{T}_{\mathrm{i}}=190 \mathrm{eV}$. By defining electron and ion collisionality parameters according to [5], Eq. (4.105) as $v_{e e}^{*} \equiv L / \lambda_{e e} \approx 10^{-16} n_{e} L / T_{e}^{2}$ and $v_{i i}^{*} \equiv L / \lambda_{i i} \approx 10^{-16} n_{e} L / T_{i}^{2}$, where $L$ is the product $\pi q_{95} R$, with $q_{95}=5$ being the safety factor at the surface containing $95 \%$ of the poloidal magnetic flux, $R=1.7 \mathrm{~m}$ - major radius of the plasma, and assuming $\mathrm{Z}_{\mathrm{eff}}=1$, one obtains $v_{e e}^{*}=4.5$ and $v_{i i}^{*}=1.3$. Hence, ions may be considered as almost collisionless and electrons - marginally collisionless in the SOL. 
Since the completion of the sensitivity studies described in [1], other checks have been made in order to assess the sensitivity of the SOLPS solution to assumptions made in EIRENE. Runs with the newer version of EIRENE (dated 1999, compared with the older version of 1996), coupled with B2.5, were performed. The peak $\mathrm{H}_{\alpha}$ radiation was reduced by $20 \%$, while the maximum electron temperature at the outer target, $T_{e, m a x}$, only moderately increased from 14.3 to $16.3 \mathrm{eV}$. The newer EIRENE version includes elastic $\mathrm{D}_{2}-\mathrm{D}^{+}$collisions and a crude model to account for vibrational states of $\mathrm{D}_{2}$. These reactions, however, could not improve the match with the experiment. Finally, in order to ensure that the perpendicular (across the B-field) mobility of neutrals is not restricted, and avoid their excessive ionisation in a narrow layer close to the strike point, the wall temperature in EIRENE was raised from the usual value of $0.1 \mathrm{eV}\left(1160^{\circ} \mathrm{K}\right)$ to 1 eV. As a result, the peak $\mathrm{H}_{\alpha}$ radiation further dropped by $9 \%$. Still, it was concluded that improvements/alterations in the neutral model alone could not achieve a significantly better match with the experiment, and kinetic effects related to low plasma collisionality which are not described correctly in the fluid code B2.5, may be important in real plasmas and considerably affect density and temperature distributions in the divertor. To avoid kinetic effects, a higher collisionality, Ohmic shot, was selected for the SOLPS modelling.

\section{Modelling of Ohmic shot}

A steady-state $2^{\text {nd }}$ phase of a standard AUG Ohmic shot, with higher density, $\bar{n}_{e}=3.65 \times 10^{19} \mathrm{~m}^{-3}$, was selected for the SOLPS modelling. The magnetic configuration for this shot with $\mathrm{B}_{\mathrm{t}}=2 \mathrm{~T}, \mathrm{I}_{\mathrm{p}}=0.8 \mathrm{MA}, q_{95}=4$ (the difference in $q_{95}$ with the H-mode shot is mainly due to the smaller size plasma), is shown in Fig. 1, together with the SOLPS numerical 
grid. The plasma density was maintained by the gas puff of $3 \times 10^{21} \mathrm{~s}^{-1}$ (in terms of D atoms), which was modelled by a poloidally uniform gas puff in EIRENE. The input power into the numerical grid, $0.52 \mathrm{MW}$, was found by subtracting radiated power in the core from the input Ohmic power. Within the grid, radiated power of at least $0.175 \mathrm{MW}$, mostly coming from the SOL and divertor, was to be matched by the modelling. Modelling assumptions are standard and described in [1]. Against original expectations, the consistency between simulated and experimental parameters in the divertor for this Ohmic shot was worse than that for the previously modelled H-mode shot, despite a much higher collisionality in the SOL, as will be shown below. This might be related to the fact that the power balance in the outer divertor is more delicate for plasmas with lower input power.

Measured and simulated upstream density and temperature profiles at the outer midplane position are shown in Fig. 2. Error bars for the experimental $n_{\mathrm{e}}$ data are $\approx 6 \%$, almost constant across the profile in the core, separatrix region and stretching up to $2 \mathrm{~cm}$ into the SOL region. For the $\mathrm{T}_{\mathrm{e}}$ data, error bars are $\approx 15 \%$ for the same regions. Transport coefficient profiles across the outer midplane used in the modelling are also presented in Fig. 2, with the relation between $\chi_{\mathrm{i}}$ and $\chi_{\mathrm{e}}$ replicating that of the earlier modelled H-mode shot [1]. SOLPS profiles shown in Fig. 2 correspond to a case without drifts and impurities. All SOLPS cases displayed mismatch between the simulated and experimental radiated power in the computational domain, with the simulated values always exceeding the experimental value of $0.175 \mathrm{MW}$ due to lower $\mathrm{T}_{\mathrm{e}}$ and higher $\mathrm{n}_{\mathrm{e}}$ in the divertor than in the experiment (see below). For the case corresponding to Fig. 2, the power radiated on deuterium along was $0.2 \mathrm{MW}$. An introduction of chemical sputtering with the sputtering yield of $1.4 \%$ (the same as used previously in [1]) raised the total radiated power 
to $0.3 \mathrm{MW}$, far above the experimental value. Therefore, to obtain more realistic $\mathrm{T}_{\mathrm{e}}$ and $\mathrm{n}_{\mathrm{e}}$ at the outer target, chemical sputtering was switched off and pure deuterium plasmas were considered.

Density and temperature profiles at the outer target are shown in Fig. 3 for a case similar to that shown in Fig. 2, with the same separatrix density, but with drifts and a slightly higher input power, 0.56 MW. Upstream profiles around the separatrix position and in the SOL are almost the same for drift and non-drift cases, for the same transport coefficients. However, deviations from the experimental profiles appear near the inner core boundary due to some parasitic fluxes in the present drifts implementation in B2.5. They occur at higher $\mathrm{T}_{\mathrm{e}}, \mathrm{T}_{\mathrm{i}}$, a matter to be dealt with in the near future. Typically, drift cases have somewhat higher outer target $T_{e}$, however, as can be seen from Fig. 3, still far below the maximal experimental values obtained from the target Langmuir probes. In particular, all SOLPS cases lack the peaking of the experimental $\mathrm{T}_{\mathrm{e}}$ profile at $\approx 20 \mathrm{eV}$. In the previous modelling of the same shot [4], such a peaking could be achieved by running drift cases with fluid neutrals, however, this result was not repeated when running cases with the coupled B2.5-EIRENE code. The simulated plasma density at the target is much higher compared to that inferred from Langmuir probes. Partly, this must be due to the under-estimate of $n_{e}$ measured by the probes. Probe data in general are not very reliable, partly due to the expected deviations of the electron distribution function from Maxwellian. For the flushmounted target probes used in AUG there is also some uncertaintly about the probes' effective area. That is why an independent assessment of the over-estimate of the simulated target $n_{e}$ is important. It can be established by comparing measured and simulated power and particle fluxes shown in Fig. 4. The integral simulated power flux to the outer target is $0.21 \mathrm{MW}$, which exceeds the flux calculated from the Langmuir probes as $8 n_{e} c_{s} T_{e}$, with $c_{s}$ being the ion sound speed, only by a factor 1.35 . At the same time, the integral simulated particle flux to the outer 
target is larger than that inferred from the ion saturation current of the Langmuir probes by a much greater factor 2.52, which implies cooler plasma with larger recycling in the divertor in simulations than in the experiment. The simulated sub-divertor neutral pressure exceeded the measured value by factor 2, consistent with the larger simulated target particle flux.

\section{Discussion}

Midplane separatrix parameters for the drift Ohmic case described above are: $n_{e}=1.3 \times 10^{19} \mathrm{~m}^{-3}, \mathrm{~T}_{\mathrm{e}}=47 \mathrm{eV}, \mathrm{T}_{\mathrm{i}}=62 \mathrm{eV}$. Using similar collisionality parameters as in the previous section, gives $v_{e e}^{*}=14.6$ and $v_{i i}^{*}=7.9$. The SOL plasma is therefore much more collisional than in the H-mode case. However, for the electrons with energies of $3-5 \mathrm{~T}_{\mathrm{e}}$, which are responsible for the bulk of the heat flux, the effective collisionality is much lower. Taking e.g. $4 \mathrm{~T}_{\mathrm{e}}$ as a characteristic value, one obtains $v_{e e_{-} 4 T e}^{*}=0.9$. Therefore, the heat-carrying electrons are only marginally collisional, indicating a possible impact of kinetic effects on divertor conditions.

The upstream plasma collisionalities in the Ohmic case are typical for the AUG operation, they can only be increased substantially in higher density plasmas with detachment, which however itself presents considerable problems for the modelling. They are also not far from those expected in ITER. According to [6], the expected separatrix $T_{e}$ in ITER is $150 \mathrm{eV}$, and $\mathrm{n}_{\mathrm{e}}$ $4 \times 10^{19} \mathrm{~m}^{-3}$. Taking into account the larger size of ITER (major radius $\mathrm{R}=6.3 \mathrm{~m}$ compared to $1.7 \mathrm{~m}$ in AUG) and lower $q_{95}$ (3 compared with 4), one obtains $v_{e e}^{*}=12.3$, which is very close to that of the modelled AUG Ohmic case $\left(v_{e e}^{*}=14.6\right)$. 
The concrete mechanism by which supra-thermal electrons or ions might raise plasma temperatures in the divertor and reduce its density is not clear at present. It is possible that a large fraction of supra-thermal particles in the divertor originating from the 'hot' SOL will raise the effective heat conduction, leading eventually to higher heat deposition onto the targets for the same upstream pressure. Under such circumstances, the application of 'flux limits' to parallel transport coefficients would be erroneous, and one should instead introduce 'flux enhancement factors', as was concluded in the kinetic simulations in [7]. Within the strategy of the edge modelling adopted in this paper, it would allow one to shift the position of the separatrix more outward (towards positive distances from the separatrix in Fig. 2). The separatrix position is poorly defined in experiment, with the uncertainty of $\approx 1 \mathrm{~cm}$ in $\mathrm{AUG}$ due mainly to the poor accuracy of its determination by the equilibrium code used in the grid generation. However, the relation between midplane $n_{e}$ and $T_{e}$ is reliable, as both quantities come from the same laser diagnostic. So, if for lower upstream parameters (namely electron pressure $n_{e} \times T_{e}$ ) it was possible to transfer more heat flux onto the target, one could select lower separatrix $n_{e}$ and still satisfy the power balance (the amount of power conducted to the target). The reduction in the separatrix $n_{e}$ might then result in a 'hotter' divertor solution, with higher $T_{e}, T_{i}$ and lower $n_{e}$ at the target (within the range of $\mathrm{n}_{\mathrm{s}}=0.6-1.3 \times 10^{19} \mathrm{~m}^{-3}$ for the midplane separatrix density, the maximum simulated target $\mathrm{T}_{\mathrm{e}}$ was found to vary roughly according to $n_{s}^{-1.5}$, for the same transport coefficients). Further modelling work, with the implementation of kinetic calculations into the present-day fluid codes, is required to test these ideas. It is, however, clear from Fig. 5 that there indeed may be a scope for some reduction in the upstream separatrix $n_{e}$ in the modelling. This figure presents the comparison between electron pressures from the SOLPS simulation and from the reciprocating probe introduced below the X-point, as shown in Fig. 1. Further reduction in 
the separatrix $n_{e}$ in the simulations would not contradict the experiment, since the outer peak of the simulated electron pressure profile is clearly above the corresponding experimental values.

\section{Summary}

A detailed comparison between simulated and experimental divertor parameters of the ASDEX Upgrade Ohmic and H-mode discharges was performed with the SOLPS (B2-EIRENE) code. It was established that the code tends to underestimate the divertor $T_{e}$ and overestimate the plasma density, thus, predicting cooler and denser divertor than in the experiment. Sensitivity checks, by changing internal input parameters of SOLPS, were made in an attempt to reach better agreement with the experiment. At present, it seems unlikely that the discrepancies could be caused by either deficiencies of the neutral Monte-Carlo package EIRENE, or its improper implementation in the cases analysed. An alternative explanation via kinetic effects capable of increasing parallel heat fluxes seems more plausible. It is important to point out that for most of the AUG plasmas heat carrying electrons near the separatrix in the main SOL (far away from the divertor) with energies of $\sim 4 \mathrm{~T}_{\mathrm{e}}$ are only weakly collisional. The same conclusion applies to the expected operational conditions of ITER. Hence, proper implementation of kinetic effects in today's fluid codes becomes an urgent task. 


\section{References}

[1] Chankin A V, Coster D P, Dux R, et al., Plasma Phys. Control. Fusion 48 (2006) 839.

[2] Schneider R, Coster D P, Kallenbach A, et al., 'Test of the Predictive Capability of B2EIRENE on ASDEX Upgrade', Proc. $17^{\text {th }}$ Int. Conf. on Plasma Physics and Controlled Fusion Research 1998 F1-CN-69/THP2/05 (Vienna: IAEA).

[3] Schneider R, Coster D P, Neuhauser J, et al., proc. of $22^{\text {nd }}$ EPS Conference on Controlled Fusion and Plasma Physics, Bournemouth, $3^{\text {rd }}-7^{\text {th }}$ July 1995, volume IV, pp. 285-288.

[4] Coster D P, Chankin A V, Conway G D, et al., proc. of $32^{\text {nd }}$ EPS Conference on Plasma Phys. Tarragona, 27 June - 1 July 2005 ECA Vol.29C, P-1.008 (2005).

[5] Stangeby P C, in The Boundary of Magnetic Fusion Devices, IOP Publishing, Bristol (2000), p.656.

[6] Kukushkin A S, Pacher H D, Pacher G W, Janeschitz G, Coster D, Loarte A and Reiter D, Nucl. Fusion 43 (2003) 716.

[7] Kukushkin A S, Runov A M, Contrib. Plasma Phys. 34 (1994) 2/3, 204. 


\section{Figure captions}

Fig. 1. Magnetic configuration and the SOLPS numerical grid for the standard AUG Ohmic shot \#18737, together with the trajectory of the reciprocating Langmuir probe.

Fig. 2. Measured and simulated density and temperature profiles near the outer midplane and transport coefficients used at the outer midplane, for the standard AUG Ohmic case.

Fig. 3. Experimental and simulated $n_{e}, T_{e}$ profiles at the outer target, simulated $T_{i}$ profile for the standard AUG Ohmic case. The SOLPS case is with drifts, but without impurities, the input power $0.56 \mathrm{MW}$ is slightly higher than in the experiment.

Fig. 4. Experimental and simulated power and particle fluxes to the outer target, for the standard AUG Ohmic shot.

Fig. 5. Electron pressures from SOLPS and the reciprocating probe introduced below the Xpoint, as shown in Fig. 1. 


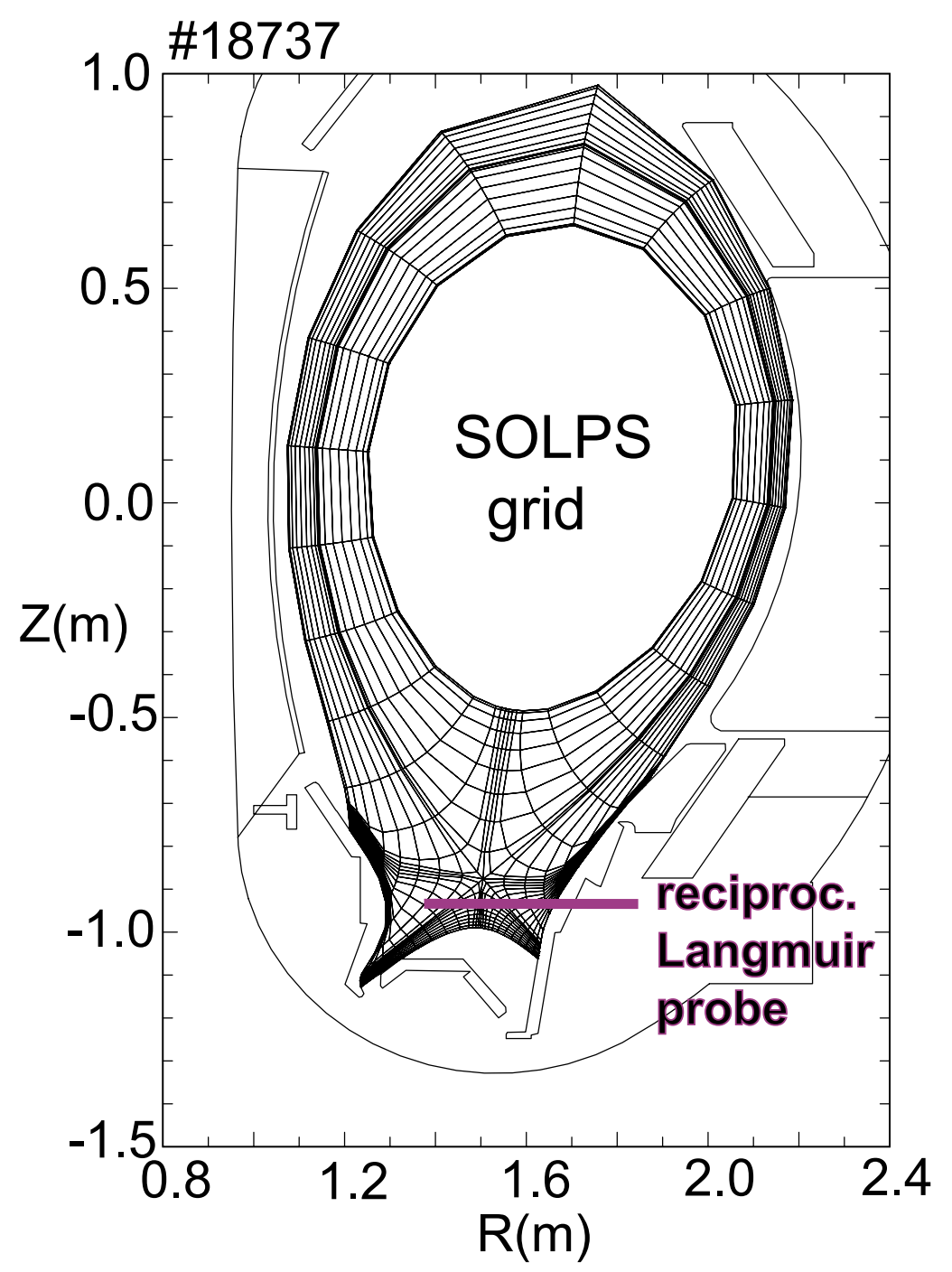

Fig. 1 


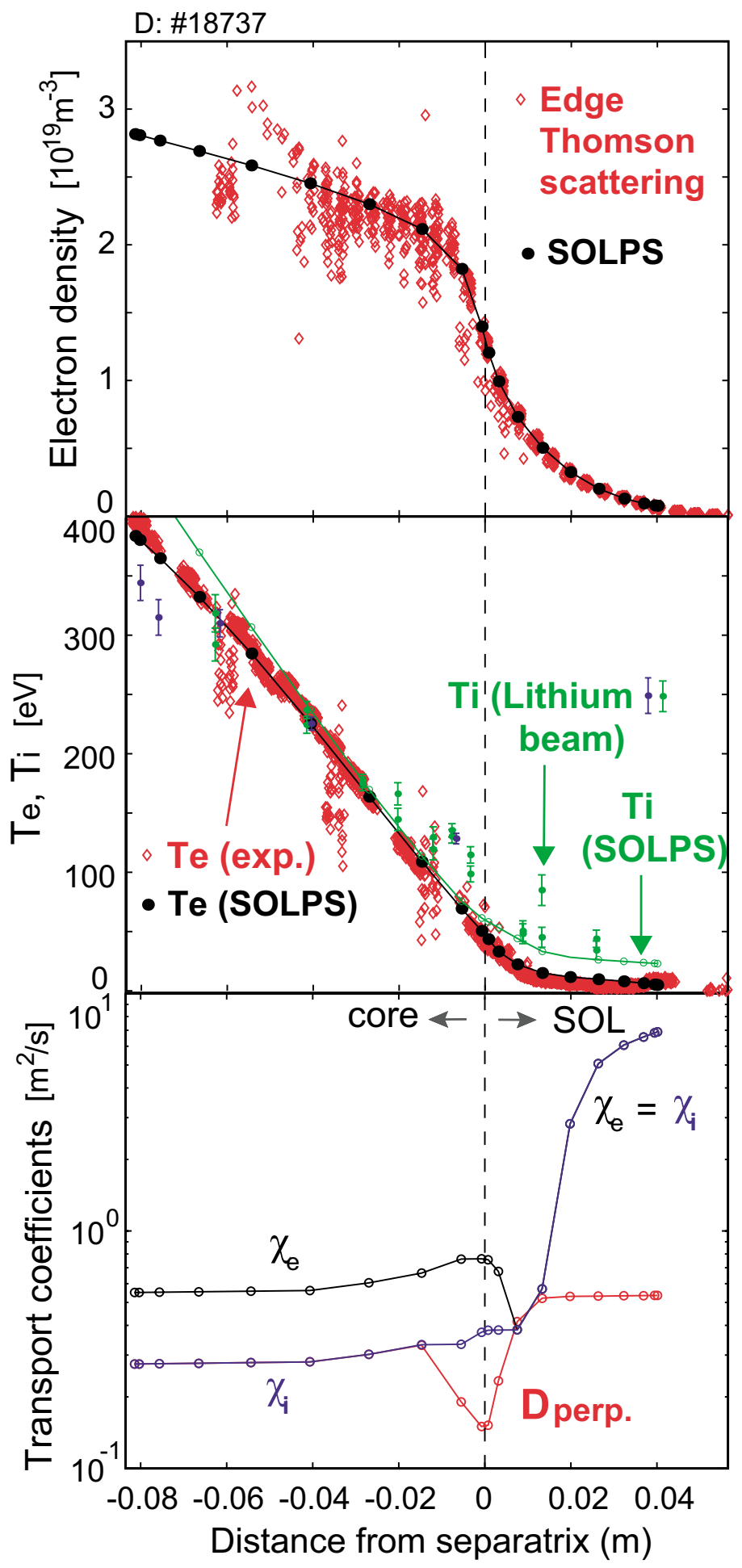

Fig. 2 

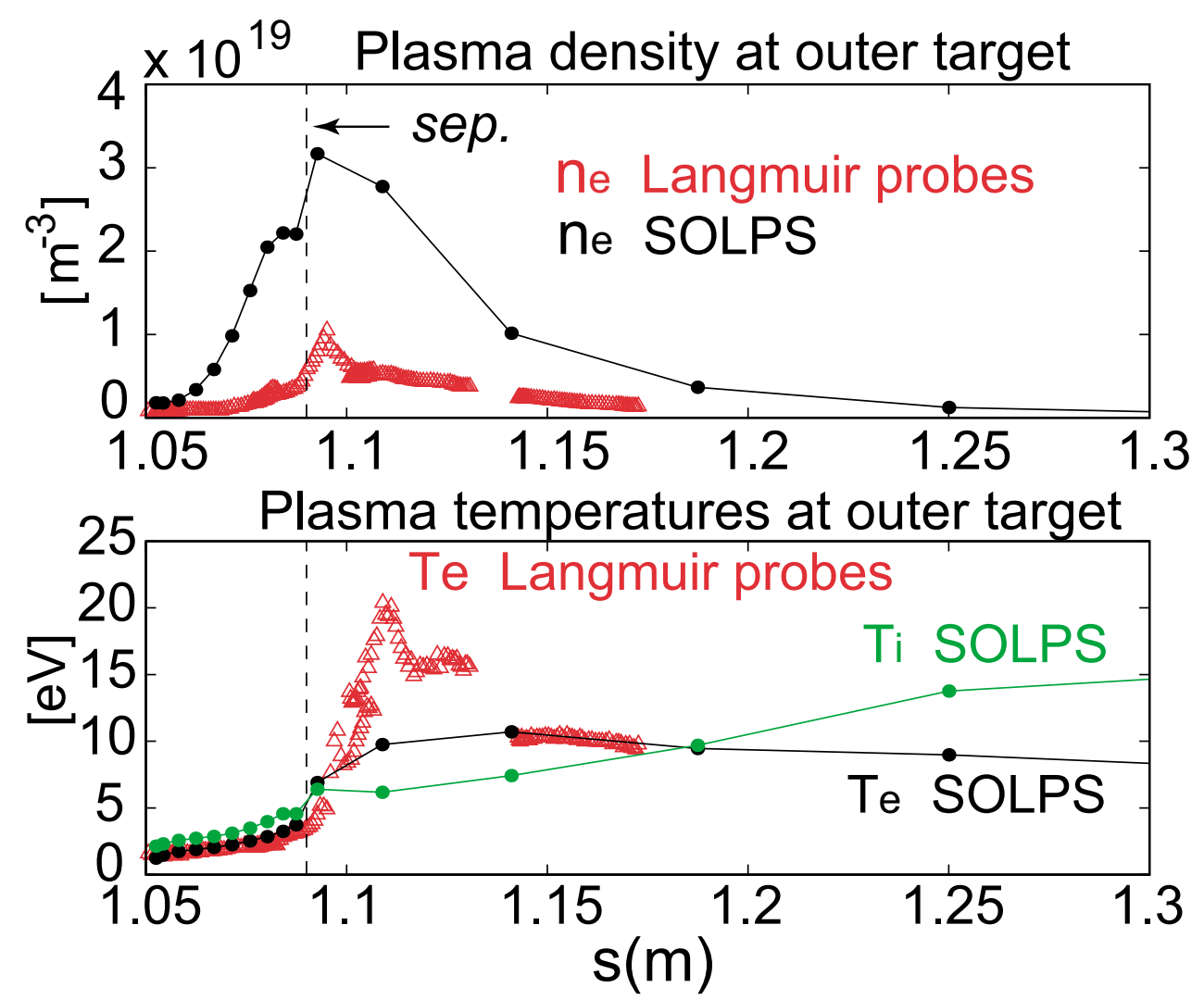

Fig. 3 

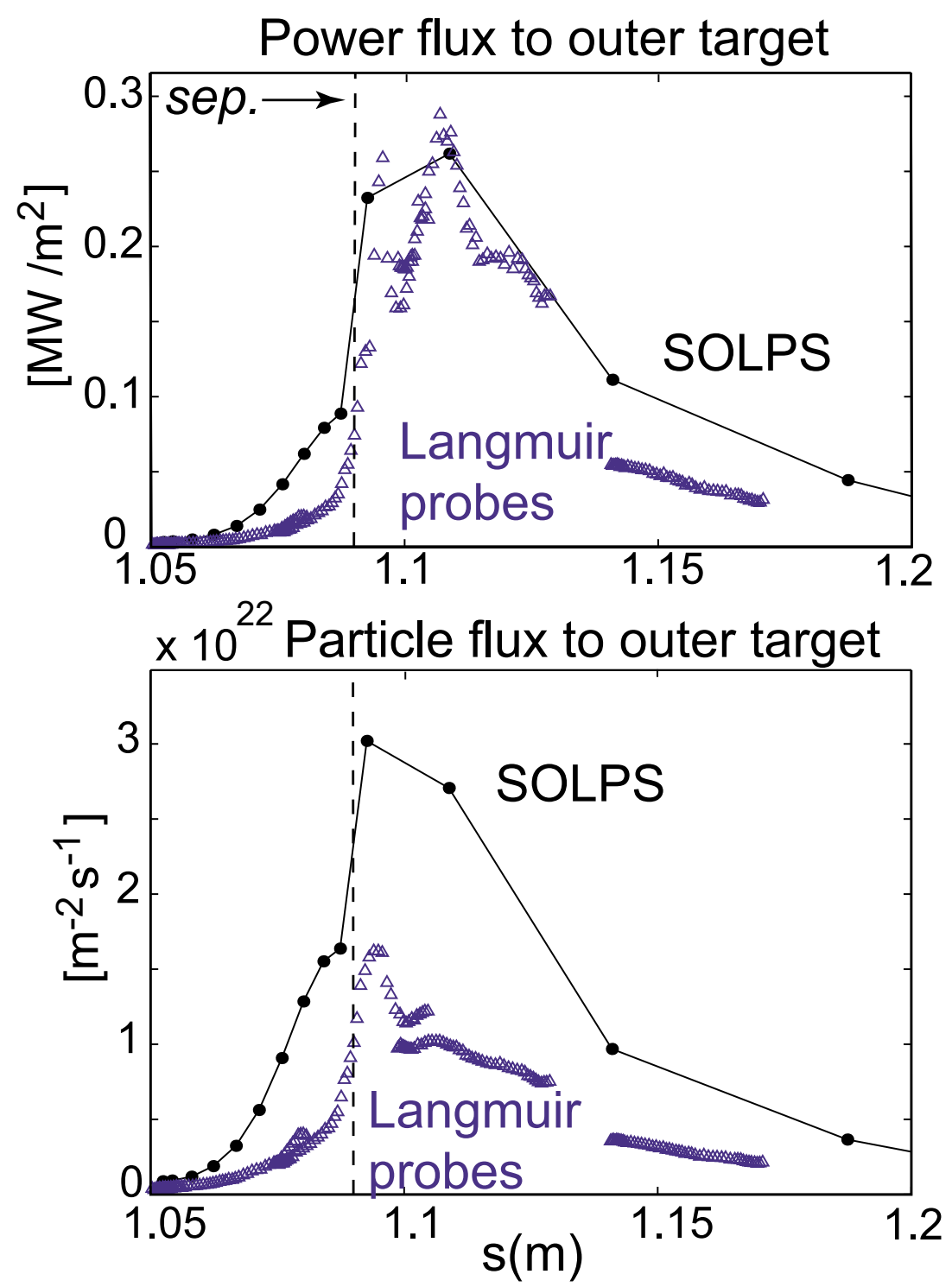

Fig. 4 


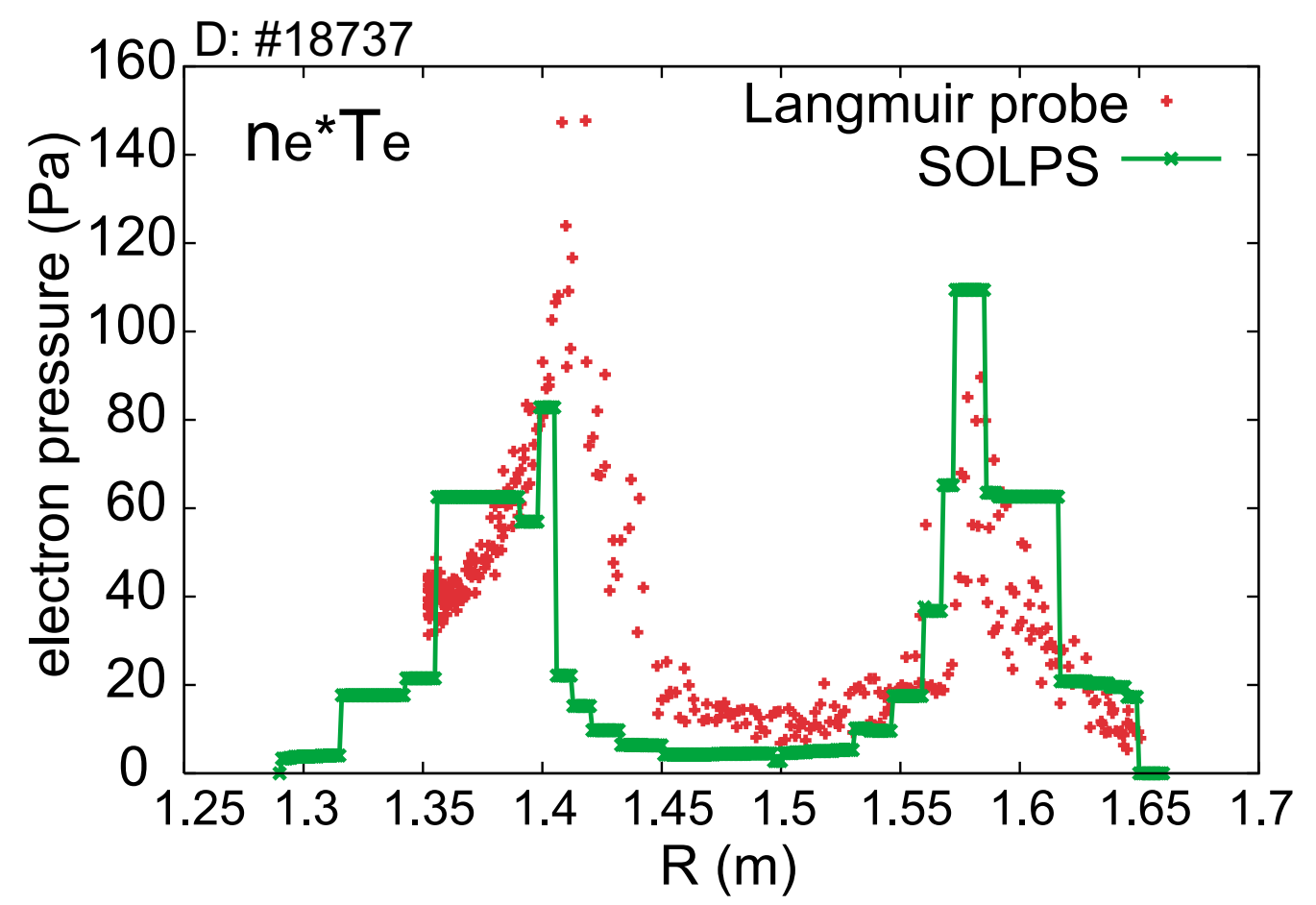

Fig. 5 